\begin{tabular}{|l|l|l||}
\hline \multicolumn{2}{|c|}{ PublisherInfo } \\
\hline \hline PublisherName & $:$ & BioMed Central \\
\hline \hline PublisherLocation & $:$ & London \\
\hline \hline PublisherImprintName & $:$ & BioMed Central \\
\hline \hline
\end{tabular}

All you need for proteomics

\begin{tabular}{|l|l|l||}
\hline \multicolumn{2}{|c|}{ ArticleInfo } \\
\hline \hline ArticleID & $:$ & 3927 \\
\hline \hline ArticleDOI & $:$ & $10.1186 /$ gb-2001-2-7-reports2004 \\
\hline \hline ArticleCitationID & $:$ & reports2004 \\
\hline \hline ArticleSequenceNumber & $:$ & 21 \\
\hline \hline ArticleCategory & $:$ & Web report \\
\hline \hline ArticleFirstPage & $:$ & 1 \\
\hline \hline ArticleLastPage & $:$ & 4 \\
\hline \hline & & RegistrationDate : 2001-5-15 \\
ArticleHistory & $:$ & Received \\
\hline ArticleCopyright & $:$ & BioMed Central Ltd2001-5-15 \\
\hline \hline ArticleGrants & $:$ & \\
\hline \hline
\end{tabular}




\begin{tabular}{|l|l|l||}
\hline ArticleContext & $:$ & 130592277 \\
\hline
\end{tabular}

\section{David Chambers}

\section{Abstract}

The ExPASy proteomics server is a dedicated site for the analysis of protein sequences, protein structures and two-dimensional polyacrylamide gel electrophoresis.

\section{Content}

The ExPASy (Expert Protein Analysis System) proteomics server is a dedicated site for the analysis of protein sequences, protein structures and two-dimensional polyacrylamide gel electrophoresis (PAGE). ExPASy is best defined as a source of, and an entry point into, all the commonly required proteomics tools and databases. There is a comprehensive set of proteomics services ranging from simple applications such as DNA translation software and protein-protein alignments to more intricate modeling systems encompassing primary, secondary and tertiary structure analysis. The site can deal with a several types of protein enquiry. It can be used to investigate the characteristics of a target aminoacid sequence systematically or to act as the starting point to access previously accumulated data on known proteins. In either case the tools available are ordered in logical fashion such that they follow an increasing level of complexity. As a result, there is a good amount of connectivity between different aspects of the site, whereby the results from one analytical tool can be used as the source of further enquiry or cross-referenced with results from related tools. Ultimately, the aim of ExPASy is to be a first port of call for all protein enquiries.

\section{Navigation}

The web interface is well laid out, with each set of tools represented by a single hyperlink. Services available are grouped according to their general function, such as protein similarity searching or secondary structure prediction. Initially, it is assumed that the user is familiar with what these services have to offer, but, the true depth of the site is revealed by following one of these links, whereupon a great deal more explanatory information is provided in conjunction with a comprehensive listing of the tools available to perform the specified task. For example, entry to the 'Transmembrane Region Detection' tool provides eight further hyperlinks, each with a brief description of the manner in which the tool serves its purpose. This hierarchy is further reinforced after arrival at a particular tool, where specific instructions can be retrieved about the application. In this way, users can select the appropriate 
way to investigate their protein without in-depth prior knowledge. The whole server is well coordinated with other dedicated proteomics sites, so that any given tool can readily be located.

\section{Reporter's comments}

\section{Timeliness}

The ExPASy sever was last updated on 18 January 2001. Additions and improvements can be viewed at the 'What's new on ExPASy' hyperlink, which also provides information on the applicability of new software.

\section{Best feature}

ExPASy's coverage of the world of proteomic analysis is its greatest strength. Many sites aim to achieve this but few are so accomplished and well ordered. For each tool there are several available programs. Although this may appear redundant, combinatorial use of related packages compensates for any given weakness in a single program. The user can very rapidly navigate the ExPASy server and be relatively well informed as to the nature of the enquiry. In addition, all of the software provided is in the public domain. So, one could obtain staggering amounts of information about a query sequence from the free services provided here. Further detailed analysis, for example three-dimensional sequence-structure predictions, would probably require intimate knowledge of dedicated software, and this is likely to involve a subscription. None of the tools provided here are particularly more advanced than those available elsewhere, but taken together they form a comprehensive resource. The ExPASy server makes up for what it cannot do by providing a hyperlink to other molecular biology servers. In this, it probably provides one of the best listings of molecular biology servers on the web today.

\section{Worst feature}

Although it is relatively straightforward to obtain data, for any given analytical tool the user must be able to interpret the results and place them in the context of the initial question. Therefore, to fully appreciate the data the user must be prepared to learn the particular nuances of the program they have used. Information is usually provided by the authors of the software but learning can be time consuming, especially when more than a few tools are used in conjunction.

\section{Wish list}


It would be particularly useful if a query sequence could be investigated in a semi-automated fashion. In essence, this would involve submitting an amino-acid sequence to a generic web page, selecting the appropriate software packages from a menu, and subsequently leaving the system to process and present the data in a summarized manner. Output could be presented graphically with regions of interest highlighted. In this way, ExPASy would be able rapidly to identify regions of significant interest, whether conserved domains or coiled regions, rather than trawling through programs individually.

\section{Related websites}

Several websites act as hosts for a broad array of molecular biology and proteomics tools and they all fulfill the principal remit of ExPASy. ExPASy is hyperlinked to the majority of these, and the others can be accessed directly from other sites. The most useful of these servers are the operated from the European Bioinformatics Institute (EBI) and Japanese GenomeNet. Some of these sites perform particular tasks more efficiently than ExPASy - for example, Japanese GenomeNet has a coordinated program for a protein motif searching package whereas ExPASy has a collection of individual tools for the same purpose - so it is worth visiting them all to before embarking on a particular job.

\section{Table of links}

ExPASy WWW server

European Bioinformatics Institute (EBI)

Japanese GenomeNet

\section{References}

1. ExPASy WWW server.

This PDF file was created after publication. 